\title{
Schlüssel zu Gefühlen
}

\author{
Kommunikation bei Demenz Beziehungen zu demenzkranken Patienten aufzubauen \\ und diese emotional zu erreichen, ist manchmal gar nicht so einfach. Wir stellen Ihnen \\ zwei unterschiedliche Ansätze vor, mit denen Sie kommunikative Brücken zu Ihren \\ Patienten bauen und so den Kontakt intensivieren können: die Musiktherapie und den \\ Hundebegleitdienst.
}

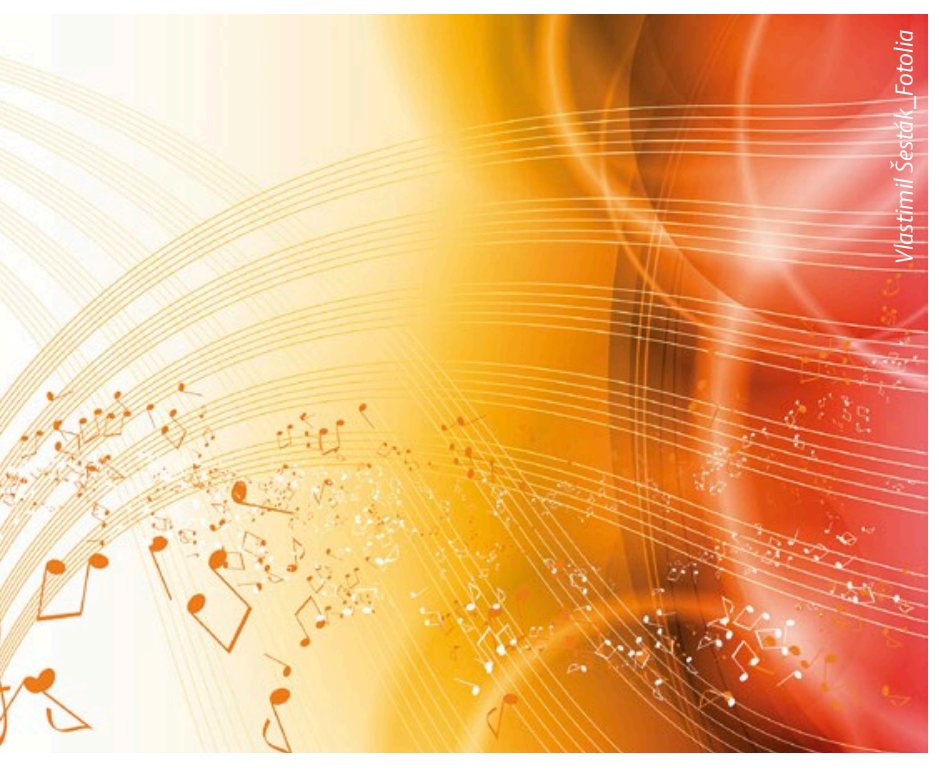

Musik wird im Umgang mit Demenzkranken zu einer Sprache, die aus der Sprachlosigkeit herausholen kann.

- Astrid McCornell -

\section{Musiltherapie: Wotan rappt in Walhalla}

1ch habe mich zum Wintersemester in der Uni Hamburg angemeldet. Das Kontalistudium für ältere Erwachsene ermöglicht ein reines Neigungsstudium und ich will meiner Neigung zur Musik etwas mehr Raum geben. Der Kurstitel "Wotan rappt in Walhalla" erscheint mir vielversprechend.

"Nun", fragt die Dozentin die Studierenden am ersten Vorlesungstag, "warum sind Sie hier, und was erwarten Sie von dem Seminar?". Ich komme dran, fange an zu erzählen, gerate in Fahrt und schwärme von der Wirksarnkeit der Musiktherapie bei demenzkranken Menschen. "Das ist ja spannend", meint die Dozentin, "halten Sie doch mal einen Vortrag über Musiktherapie". Das habe ich nun von meiner Schwatzhaftigkeit.
Ich beschliepe, eine mir bekannte Musilktherapeutin aufzusuchen und mir die Sache genau erklären zu lassen. Bis dato sah für mich Musiktherapie nach gemeinsamem Singen und Musizieren aus, und das therapentische Ziel war die daraus gewonnene Lebensfreude und Selbstbestätigung. Die Musiltherapeutin Inga Auch-Johannes empfängt mich in ihrem Arbeitszimmer. Das Zimmer ist angefüllt mit den unterschiedichsten Musikinstrumenten und so fühle ich mich gleich wohl.

"lch habe eine Frage von der Dozentin mitgebracht", beginne ich unser Gespräch, "welche Musik wird in der Musiktherapie verwendet - und warum?" "Also", erwidert Inga Auch-Johannes, "es gibt keine passende Musik gegen Depressionen. So funktioniert das nicht".

Dass Musik heilende Wirkung hat, wird schon im Alten Testarment berichtet. Da heilt David mit der Musik seiner Harfe den König Saul, welcher von einem bösen Geist heimgesucht wurde. In der Musiltherapie gibt es verschiedene Strömungen, so gibt es Therapeuten, die sich zum Beispiel nur mit Schmerzredultion beschäftigen.

Doch die qualifizierte Musiktherapie versteht sich als tiefenpsychologische Therapie, deren Aufgabe der Aufbau einer Beziehung und das Wiederherstellen der Gesundheit ist.

Musiltherapie wird zum Beispiel bei Ängsten, psychiatrischen Krankheiten, Demenz, neurologischen Erkrankungen und Entwicklungs- und Verhaltensstörungen eingesetzt. Dabei werden zwei Behandlungsfelder unterschieden: die rezeptive und die alkive Musiktherapie. Bei der rezeptiven Musiktherapie wird gemeinsam Musik gehört, bei der ahtiven Musiktherapie wird gemeinsam musiziert.

"Muss man ein Instrument spielen können, um an der Musiktherapie teilzunehmen?" frage ich Inga Auch-Johannes. 
"Absolut nicht", beruhigt sie mich, "ich erkläre es dir anhand eines Beispiels".

"Zu mir kommt Herr Schulz. Herr Schulz ist von seinem Psychiater geschickt worden. Er leidet schon lange an Depressionen, und hat sämtliche Therapien ohne Erfolg absolviert. Ob Gesprächs-, Verhaltens- oder Ergotherapie, Medikamente und Krankenhausaufenthalt, nichts hat geholfen. Musiktherapie als letzter Versuch.

Herr Schulz kommt also ins Musiktherapie-Zimmer. Er schaut sich misstrauisch um und erklärt gleich, dass er auf keinen Fall Musik mit mir machen möchte. So was Albernes hat er noch nie gemacht. Also beginne ich ein Gespräch und biete Herrn Schulz vielleicht einfach mal ein Instrument an. Zu Beispiel die Bordum Kantile."

Mit diesen Worten legt Inga Auch-Johannes mir ein wunderschönes Saiteninstrument auf den Schoss. Ich schlage die Saiten sanft an und es ertönt einen warmen Klang. Das Instrument vibriert in der Tiefe seines Holzkörpers. Ich bin entzücht. Nicht aber unser filktiver Herr Schulz.

Inga Auch-Johannes erzählt weiter: "Herr Schulz schaut die Kantile an und beginnt über deren Verarbeitung zu sprechen. Über das Holz und die Leimung. Anschlagen will er sie nicht. Ich lasse ihn in Ruhe das Instrument erkunden, beobachte ihn dabei genau. Undich steige in das Gespräch über die Holzverarbeitung ein. So beginne ich langsam, eine Beziehung zu Hern Schulz aufzubauen.

In der nächsten Stunde bekommt Herr Schulz von mir ein weiteres Instrument angeboten, zum Beispiel lade ich ihn zum gemeinsamen Klavierspiel ein. Vielleicht wird er wütend auf das Klavier härnmern, aber dann hat er endich wieder ein Gefühl gezeigt. Wut.

Und so kommen wir in die Kommurikation über Wut. In den nächsten Wochen finde ich weiteren Zugang zu den verschütteten Gefühlen von Herrn Schulz. Er kann nun beginnen, sein Leben wieder selbstbestirnmt in die Hand zu nehmen."

Inga Auch-Johannes nennt mir ein weiteres Beispiel, wie Beziehungsgestaltung in der Musiltherapie geschehen kann. "Frau Michel wird zu Hause gepflegt. Sie lebt bei ihrem Sohn und ist $81 \mathrm{Jahre}$ alt. Als ich zu meinem Hausbesuch komme, finde ich sie wütend vor. Kch frage den pflegenden Sohn,

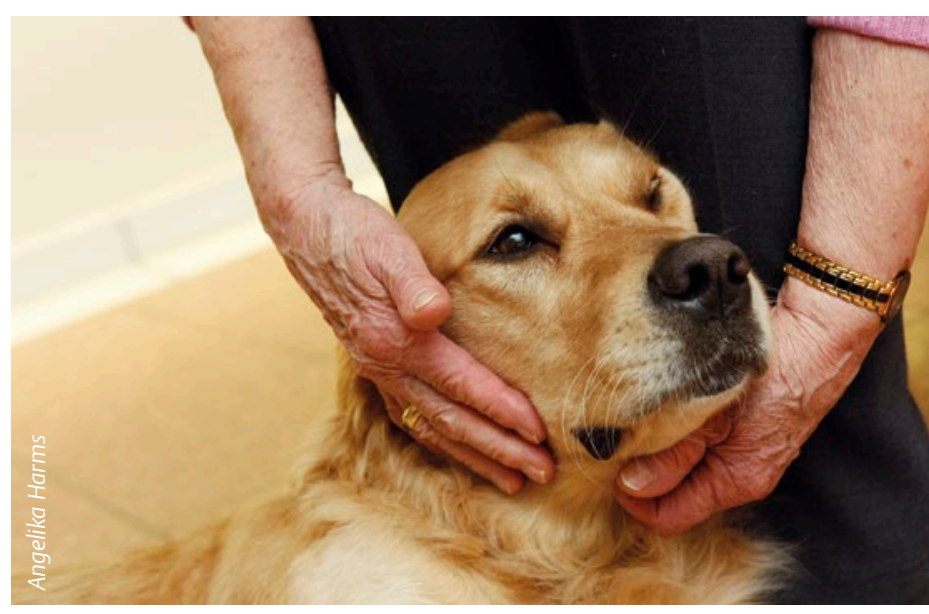

Hunde sind eine wunderbare Kommunikationsbrücke in die Welt der Demenz.

ob er einen Grund für die Stimmung der Mutter angeben kann. Heute ging schon alles irgendwie schief, ich weip auch nicht warum', antwortet er.

Frau Michels Wut äupert sich in Schimpten und Trampeln, zwischendurch sind ihr trauriger Blick und ihre Erschöpfung zu sehen. lch setzte mich Frau Michel gegenüber und beginne, ihr Schimpten rhythmisch zu begleiten und das Trampeln mit wenigen Trommelschlägen zu akzentuieren. Da bei Frau Michel kein Text zu verstehen ist, derke ich mir zu meiner erfundenen Musik zunächst nur Ausrufe aus wie, $O$ h, nein!' und, Wie schrecklich!'.

Aus den musikalischen Motiven formt sich dann eine Melodie und aus den Ausrufen wird allmählich ein kleiner Text. In Frau Michels Alktionspausen passt sich die Melodie der veränderten Stimmung an. Nach einer Weile blickt Frau Michel auf. kh wiederhole noch einmal das kleine Lied und berühre am Ende sanft Frau Michels Arm."

Dieses Beispiel findet sich in Inga Auch-Johannes Buch "Klangbrücken - Musiltherapie in der häuslichen Versorgung für Menschen mit Demenz - ein Leitfaden für die Praxis", welches sie gemeinsam mit Prof. Dr. Eckard Weymann herausgegeben hat.

"Musik ist der Schlüssel zu den Gefühlen der Menschen, durch gemeinsames Improvisieren auf den Instrumenten werden die Gefühle gezeigt, von mir aufgenommen, unterstützt oder langsam verändert. So kann die Melodie des Klavieres von wütend zu traurig umschwingen und zu hoffnungsvoll. Auf diese Weise wird in der Musiltherapie behandelt," erklärt mir Inga Auch Johannes. "Das ist ja wie Validation", ist das Erste, was mir einfällt. 
Die Entwicklerin der Validation, Naomi Feil, nutzt ganz ähnliche Herangehensweisen, um Menschen mit Demenz das Ausdrücken und Verarbeiten ihrer Gefühle zu ermöglichen. "Jemanden zu validieren bedeutet, seine Gefühle anzuerkennen, ihm zu sagen, dass seine Gefühle wahr sind", ist die kürzeste Erklärung für Validation.

Zu den Grundsätzen der Validation gehört unter anderen: Schmerzliche Gefühle, die ausgedrücht, anerkannt und von einer vertrauten Person validiert werden, werden schwächer. Schmerzliche Gefühle, die man ignoriert und unterdrücht, werden stärker. Einfühlung und Mitgefühl führen zu Vertrauen, verringern Angstzustände und stellen die Würde wieder her. Genau diese Empathie, die es braucht um die Gefühle des anderen wahrzunehmen und auszudrücken, zeigt Inga Auch-Johannes in ihrer Musiktherapie.

Nun habe ich verstanden: Musile wird zu einer Sprache, die aus der Spracklosigkeit herausholen kann. Dass dies auch sehr gut mit demenzkranken Menschen funktioniert, kann ich mir jetzt vorstellen.

\section{Was mackt eigentlich ein \\ Hundebegleitdienst? Glücklich!}

Kennengelernt habe ich den Hundebegleitdienst schon vor über einem Jahn, als ich begann, ehrenarntliche Helfer in der Demenzbetreaung zu unterrichten. Thema des Unterrichts ist die Kommunikation mit demenzkranken Menschen und die damit verbundenen Tücken.

In meinem Unterricht sapen Hundebesitzer, die sich ehrenarntlich engagieren. Sie sollen in Zukunft mit ihren Hunden Demenzhrarke besuchen, einzeln oder auch in Gruppen.

Ich selbst hatte einmal ein sensationelles Erlebris mit einem Beagle und einer demenzkranken Darne. Die Dame lag im Pflegebett, zeigte wenig Eigenbewegung, kein Interesse an ihrer Umwelt und sprach kein einziges Wort mit mir. Ich habe sie regelmäpig ein Jahr lang gesehen, und sie hat ME mit mir gesprochen. Ich war überzeugt, dass sie ihre Sprachfähigkeit verloren hatte.

Bis zu dem Tag, an dem die Haushaltshilfe in Begleitung eines Beagles erschien. Der Hund lief gleich schwanzwedelnd auf die Darne zu, diese richtete sich in ihrem Pflegebett auf und sprach zu dem Hund: "Na, mein Süper, willst au ein Leckeri?". Dabei
Wlopfte sie einladend mit der Hand auf ihr Bett. Glauben Sie mir, ich war sprachlos! So etwas hatte ich noch nie erlebt!

Dementsprechend neugierig war ich, die Erfahrungen der Hundebesitzer aus meinem Unterricht zu hören. Diese waren durchweg positiv und so wurde ich eingeladen, an einem Besuch der Betreuungsgruppe für demenzhranke Menschen teizunehmen.

Die beiden Therapiehunde "Barni" und "Lizzy" sollten die Betreaungsrunde besuchen. Kh komme rechtzeitig an, um erst einmal am Kaffeetrinken teizunehmen. Das gibt mir die Gelegenheit, die vier Damen mit Demenz ein wenig genauer anzuschauen und wahrzunehmen. Jede der Damen hat eine persönliche Begleitung an ihrer Seite.

Ich möchte lhnen die Damen kurz vorstellen:

Frau R. sitzt im Rollstuhl, sie wirkt ein wenig schläfrig, ihre Augen sind halb geschlossen und sie bewegt sich wenig. Sie bekommt einen Trinkhalm in ihren Kaffeebecher, die Sahnetorte wird ild angereicht und schwupp, hat sie sich schon verschlucht. Sie bekommt einen bösen Hustenanfall, die Helfer kürnmern sich rührend, es wird geklopft und gewischt. Erschöpft sinkt Frau R. in ihren Rollstuhl zurück, ihre Stimme flüstert einen leisen Dark. Sie wirkt sehr schwach. Frau L. ist körperich noch fit. Hübsch zurechtgemacht, mit unallrotem Lippenstift sitzt sie an der Kaffeetafel. Sie zaubert ein Bild aus ihrer Handtasche, es zeigt eine Aufnahme von ihr und den beiden Therapiehunden. "Vom letzten Mal", lackt sie und zeigt mir und allen anderen Sitznachbarn das Bild. Das wiederholt sich alle fünf Minuten, ergänzt wird das Ganze durch eine recht alte spanische Uilaubskarte.

Frau S. ist eine ältere Dame mit reizenden Silberlöckchen. Sie verputzt munter ihre Sahnetorte, antwortet höflich auf Nachfragen, beteiligt sich aber wenig an den Gesprächen am Tisch.

Frau B. ist die vierte Darne in der Runde. Sie start mich die ganze Zeit böse an, ihre Mirnik ist erstart. Ich versuche es mit der Allzweckwaffe "Lächeln", aber es kommt keine Reaktion. Sie beginnt sehr langsam den Kuchen zu essen, alles wirkt wie in Zeittupe. Sie nimmt die Kaffeetasse, ihre Hand zittert. Frau B. hat vermutich eine Lewy-Köperchen-Demenz, welche von Parkinson-Symptomen geprägt ist. Nun verstehe ich auch die fehlende Mirnik und den "bösen Blick". Auch sie spricht sehr wenig, antwortet sehr langsam auf gestellte Fragen. 
Es werden Grüpe ausgerichtet, Fotos herumgereicht und endich ist es soweit: Die Therapiehunde kommen!

Schon beim Anblick der Tiere gibt es ein aufgeregtes "Ah" und "Oh". Die Hunde laufen gleich auf die Damen zu und freuen sich offensichtlich genauso. Ein Gefäp mit HundeLeckerli wird geöffnet und an alle vier Damen und die Begleipersonen verteilt.

Die Hunde schnüffeln sich sogleich an den Rollstuhl von Frau R. heran. Dieser ist besonders interessant, wegen der heruntergefallenen Kuchenkrürnel. Frau R. fängt an zu lachen, als die Hundeschnauzen um sie herum kitzeln. Sie öffnet die Augen weit, reicht ihnen Leckerlies an, beobachlet die Hunde ganz genau, lächelt und lacht, sie kann gar nicht mehr aufhören damit. Sie hält den Hund fest, drückt und streichelt ihn.

Ich frage Frau R., ob sie früher selbst einen Hund hatte. "Nein", haucht sie. Oder ein anderes Haustier? "Nein", haucht sie erneut. lch frage sie, ob die Liebe zu den Hunden aus ihrem Herzen kommt und zeige dabei auf mein Herz. "Ja", haucht sie zurück, und hält mit dem Hund Pfötchen. Ein voller Erfolg!

Frau L., das merkt man gleich, ist ein echter Hundefan! Sie füttert und streichelt routiniert, spricht mit den Hunden und strahlt über das ganze Gesicht. Auf meine Frage nach dem eigenen Hund antwortet sie: "Ja, ich hatte einen, der sah aus wie ein Schäferhund mit einem Kringe/schwanz. Ich komme nur wegen der Hunde her." Mit diesen Worten lässt sie mich stehen, sie hat wichtigeres zu tun als zu klönen, und begibt sich auf der Suche nach dem Leckeri-Gefäp.

Auch Frau S. ist schwer beschäftigt. Sie füttert und streichelt, klopft und redet mit den Hunden. Die Hunde sind begeistert, einer wirft sich ihr zu Füpen, auf den Rücken. Sie beugt sich tief hinab, lacht und knuddelt ihn. Auf meine Nachfrage nach dem eigenen Hund, beginnt sie mir aus ihrer Lebensgeschichte zu erzählen. Dass sie mit dem Ehemann einen Hund hatte, aber jetzt bei den Kindern lebt, die haben einen blinden So hn und auch einen Hund, welche Rasse, das sei ihr grade entfallen. Das Ganze kommt etwas unsortiert, aber gut verständlich an.

Toll, denke ich, mit diesen biografischen Daten könnte man eine Din-A4-Seite füllen, viele wichtige Informationen, um sich in die Lebenswelt von Frau S. hineinzuversetzen.
Hunde sind mit allen Sinnen erlebbar und aktivieren demenzkranke Menschen.

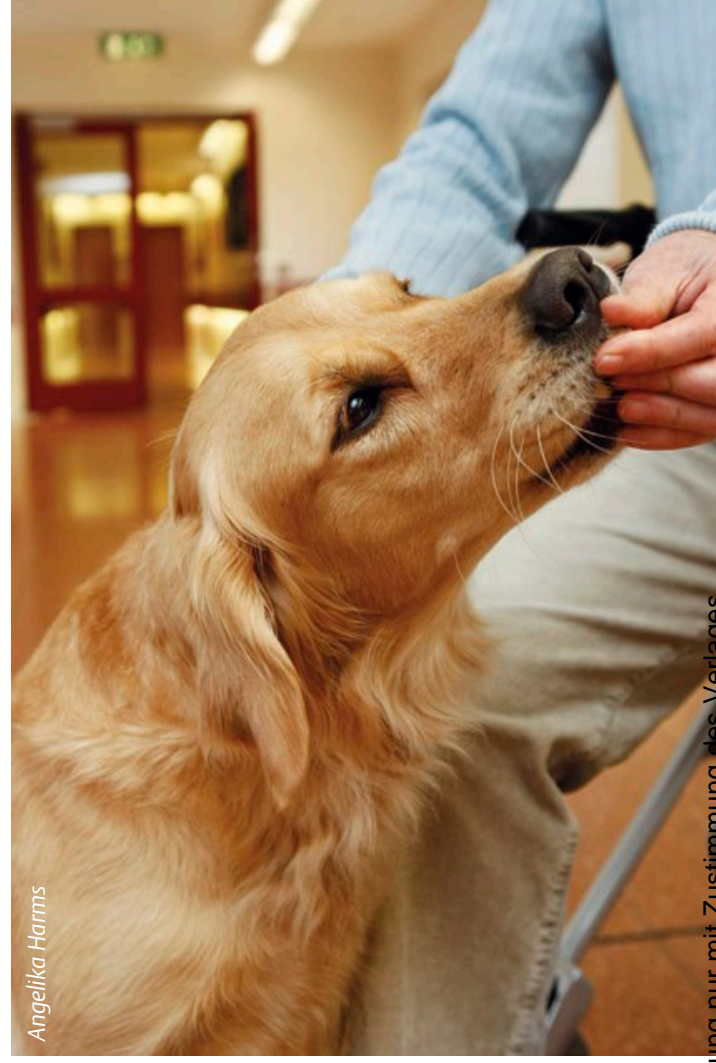

Ich schaue zu Frau B. hinüber. In ihrem Gesicht arbeitet es ordentlich, vor allem um die Mundpartie. Das Lächeln will nicht gelingen, aber ihr Blick ist wach und aufmerksam. Leckerlies in beiden Händen, die Hundeschnauzen kizzeln, Frau B. lacht, wunderbar! Auf meine Frage nach einem Haustier erzählt sie mir von ihrem grau-weipen Wellensittich. Als alle Leckerlies verteilt sind, kehrt langsam Ruhe ein. Die Hunde legen sich zu Füpen ab. Ich schaue in die Runde, alle anwesenden Damen strahlen glücklich und zufrieden!

Ich bin auch zufrieden, denn meine Erfahrungen haben sich bestätigt: Hunde sind eine wunderbare Kommunikationsbrücke in die Welt der Demenz. Sie sind mit allen Sinnen erlebbar und aktivieren die Menschen in jeder Hinsicht.

\section{Autorin}

Astrid McCornell

25 Jahre in der Pflege und im Management beschäftigt; seit 2013 selbständig als Autorin, Fotografin und Vortragsrednerin; Autorin der Bücher „Wo sich Huhn und Eule gute Nacht sagen“ und „Wenn Huhn und Eule in die Ferne schweifen“, erschienen im Ernst Reinhardt Verlag; weitere Berichte und Reportagen veröffentlicht sie in ihrem Blog www.toleranz-manufaktur/blog

E-Mail: info@toleranz-manufaktur.com

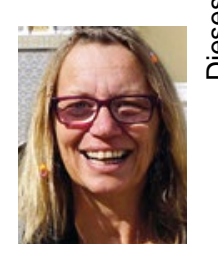

\section{Bibliografie}

DOI 10.1055/s-0042-124444

PPH 2017; 23: 80-83

(c) Georg Thieme Verlag KG

Stuttgart · New York · ISSN 0949-1619 